\title{
Capacitive sensor for the detection of residual quantity of intravenous drip solution in a plastic intravenous bag
}

\author{
Qun Wei, Sang Hyo Woo, Zia Mohy-Ud-Din*, Dong Wook Kim, Chul Ho Won**, and Jin Ho Cho ${ }^{\dagger}$
}

Abstract

Intravenous(IV) drip therapy is extensively used for all kinds of treatments. It works by delivering medicine directly into the vein. When the medicine has been fully dispensed, a dangerous situation occurs since air in the IV drip bag could enter the patient's vein, which is hazardous to the patient's safety. In this paper, using capacitive sensors to detect the residual quantity of a plastic IV drip pack is proposed. A simulation model of this technology was shown by a finite elements analysis(FEA) program to find out its feasibility and analyze the proper geometrical dimension of a capacitive sensor. According to the FEA simulation, 3 kinds of capacitive sensors were attached to the bottom surface of the plastic IV drip bag to detect the residual quantity in the experiment. Experimental data showed an agreement with the FEA simulation model estimation and confirmed that the sensor works.

Key Words : capacitive sensor, plastic intravenous drip bag

\section{Introduction}

Intravenous(IV) drip therapy is commonly used for treatments, and it works by delivering the liquids directly into the vein. It can be intermittent or continuous; continuous administration is called an intravenous drip. Compared with other routes of administration, the intravenous route is the fastest way to deliver medicine throughout the body ${ }^{[1]}$. A standard IV drip set consists of a pre-filled sterile container of medicine with an attached drip chamber which allows the fluid to flow a drop at a time. IV drips are usually made with a plastic bag in the hospital. However, there is a problem with IV drips. When no body is observing, it is impossible to know the amount of medicine delivered in a given time. Therefore the nurse needs to check the medicine while the IV drip is attached. Usually the patients indicate the remaining amount of the medicine to the nurse, but patients in intensive care are not able to alert the nurses by themselves. If the IV drip is empty for a long

School of Electronic Engineering and Computer Science, Kyungpook National University

*Dept. of Medical \& Biological Engineering, School of Medicine, Kyungpook National University

**School of Control \& Electrical Engineering, Kyungil University

'Corresponding author: jhcho@ee.knu.ac.kr

(Received : January 18, 2010, Revised : April 19, June 4, June 21, 2010, Accepted : June 21, 2010) time, air could enter into the patient's vein and possibly produce an air embolism, which would endanger the patient's safety ${ }^{[2]}$.

Y. S. Yang and H. C. Kim have discussed in their paper about a miniaturized IV drip rate meter using optical sensors to detect the fluid rate of the IV drip with an implemented system ${ }^{[3]}$. They have conducted experiments and proposed a fuzzy rule based on detection algorithms. The drawbacks of their system were that they did not focus on the residual quantity of the plastic bag IV drip detection, and their system was very complex. Recently, capacitive sensor technology has been used as a liquid volume detector. The capacitive sensor has a simple structure, compact size, and low $\operatorname{cost}^{[4]}$. The capacitive sensors can detect almost all materials and are less expensive than the optical method.

In this paper, a method to detect the residual quantity of plastic bag IV drip using capacitive sensors is proposed. A finite element analysis(FEA) simulation mode was demonstrated to estimate the feasibility and analyze proper geometrical dimensions of the capacitive sensor. According to the results of FEA simulation, the produced capacitive sensor was attached to the bottom surface of the plastic IV drip bag to detect the residual quantity. 


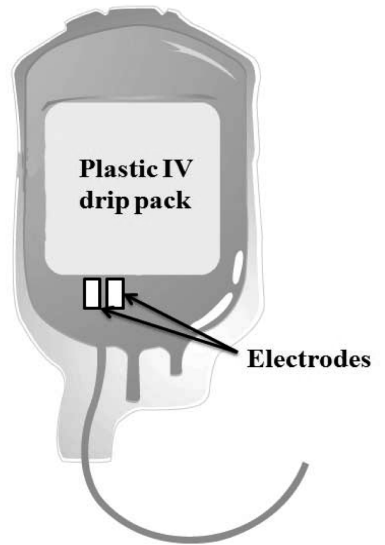

(a)

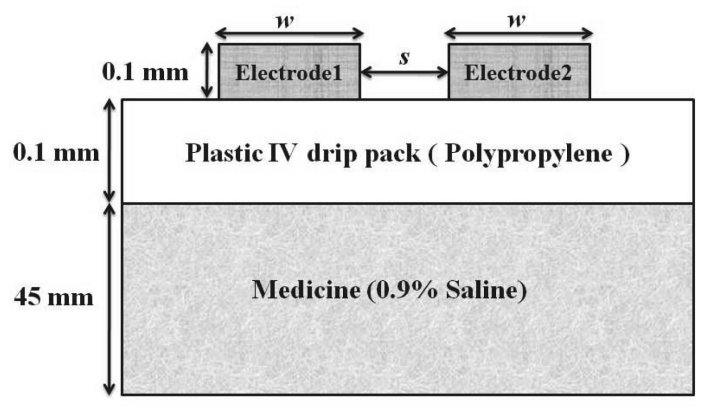

(b)

Fig. 1. The model of the capacitive sensor attached to the bottom surface of the plastic bag IV drip. (a) A front view of using a capacitive sensor to detect the residual quantity of plastic bag IV drip. (b) A cross-sectional view of a coplanar capacitive sensor over a layered material.

\section{Method}

\subsection{Capacitive sensor modeling}

Fig. 1(a) shows the basic idea of using capacitive sensor to detect the residual quantity of plastic bag IV drip: two electrodes of capacitive sensors attached to the bottom surface of the plastic bag. The plastic bag is made of polypropylene that is used in a wide variety of applications, including packaging, plastic parts, and reusable containers of various types. The two sides of the plastic IV drip bag are symmetrical and the surface is nearly flat. Therefore, the capacitive sensor is designed as a coplanar capacitive sensor, it has a benefit that the sensor's electrodes lie on the same plane. Thus, they can be applied to the material under study from one side only, instead of having to sandwich the material in between the electrodes ${ }^{[5]}$. In this study, a saline solution is used in the proposed medicine in the plastic bag IV drips. The saline solution is a general term referring to a sterile solution of sodium chloride in water and $0.9 \%$ saline is used in most of the treatment ${ }^{[6]}$. Fig. 1(b) shows a coplanar capacitive sensor over a layered medium in cross-sectional view.

\subsection{Finite element analysis}

A 2D model is proposed for the capacitive sensor because the electric field vector has only two directions from two electrodes of capacitive sensor. The model in $2 \mathrm{D}$ can ignore the fringe field effects at the outer edges of the electrodes that 3D modeling would show. The proposed model is created in Maxwell 2D $\mathrm{D}^{[7]}$. The Maxwell $2 \mathrm{D}$ program is a commercially available finite element analysis(FEA) simulation software. The problem can be solved in a 2D electro static environment due to the fact that the electrostatic potential is not rotational. A part of the Maxwell equation is shown as,

$$
\begin{aligned}
& E \cdot(x, y)=0 \\
& D \cdot(x, y)=\rho \\
& D=\varepsilon E
\end{aligned}
$$

where $E$ is the electrical potential, $D$ is the electric flux density and $\varepsilon$ is the relative permittivity. According to the vector identities equation, if the rotation angle of a vector potential is zero, then this vector potential can be shown as a gradient of a scalar quantity potential.

$$
\begin{aligned}
& E=-\nabla \phi(x, y) \\
& \nabla \cdot\left(\varepsilon_{0} \varepsilon_{r} \nabla \phi(x, y)\right)=-\rho
\end{aligned}
$$

where $\varepsilon_{0}$ is the permittivity of the vacuum, $\varepsilon_{r}$ is the relative permittivity, $\phi(x, y)$ is the electro- static potential and $\rho$ is the charge density. The capacitance represents electrostatic energy stored in electric field, and the electric field energy $U$ is determined as,

$$
U=\frac{1}{2} C V_{v}^{2}
$$

where $V_{v}$ is the voltage across the electrodes of the capacitive sensor. The electric field energy $U$ can be 
expressed in a volume integral form as,

$$
U=\frac{1}{2} \int_{V} \mathrm{D} \cdot \mathrm{EdV}
$$

Thus the capacitance $\mathrm{C}$ can be computed by the following equation:

$$
C=\frac{2 U}{V_{v}^{2}}
$$

The proposed coplanar capacitive sensor with plastic IV drip bag $(500 \mathrm{~mL} 0.9 \%$ saline $)$ in a cross-sectional view is drawn as shown in Fig. 1(b). The electrodes of coplanar capacitive sensor with the thickness fixed at $0.1 \mathrm{~mm}$. The plastic bag was $80 \mathrm{~mm}$ wide and $0.1 \mathrm{~mm}$ thick, the dimensions of the medicine-filled area had the same width as the plastic bag with the depth of $45 \mathrm{~mm}$. The electrodes of the capacitive sensor are made with copper with relative permittivity of the copper set at 1 . The polypropylene for the plastic IV drip bag was set at 2.5 . The relative permittivity of $0.9 \%$ saline was set at $75.3^{[8]}$ and conductivity was set at $1.66 \mathrm{~S} / \mathrm{m}^{[9]}$. In order to isolate the model from other voltage or charge sources the boundary conditions for the simulated space acted as a charge balloon. Excitations were set to positive $1.0 \mathrm{~V}$ and negative $1.0 \mathrm{~V}$ for each electrode to create a potential difference between the two electrodes.

In this simulation, the properties of the plastic IV drip bag in medicine and air were fixed to constant conditions, while the electrode width $(w)$ and the $\operatorname{spacing}(s)$ between the two electrodes changed and all the electrode lengths were chosen at $10 \mathrm{~mm}$. The maximum error of the solution was set at $0.01 \%$. The electric field distribution obtained from the FEA simulation is shown in Fig. 2.

Fig. 3. Shows the capacitance generated with different conditions of the capacitive sensor to detect the plastic bag IV drip filled with medicine. As shown in Fig. 3(a), the capacitance increases when the electrode width is increased. The longest one $(7 \mathrm{~mm}$ width $(w))$ shows the best effect in this simulation. And Fig. 2(b) shows that the capacitance decreases when the spacing width of the $7 \mathrm{~mm}$ between the two electrodes is increased.

\subsection{Sensor design}

As the results of the FEA simulation, the spacing between two electrodes was designed to be $1 \mathrm{~mm}$, and the width $(w)$ was designed to be 3,5 or $7 \mathrm{~mm}$ for each

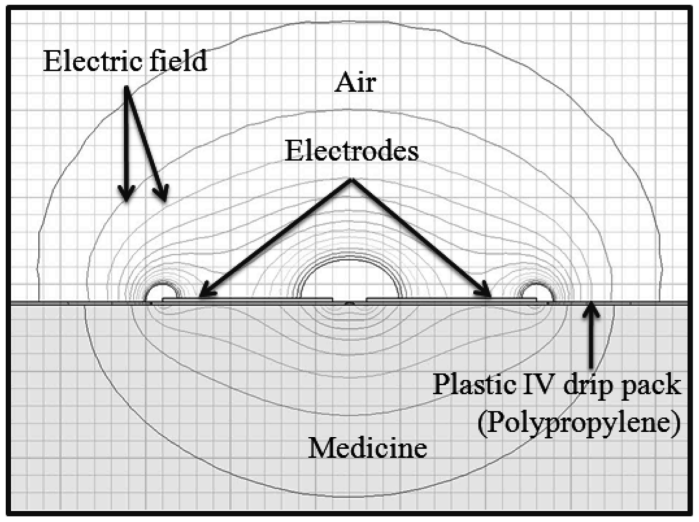

(a)

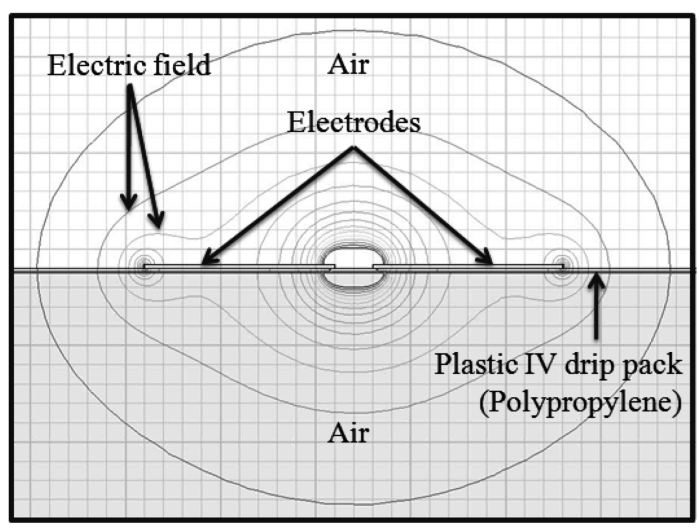

(b)

Fig. 2. The electric field obtained and from the FEA simulation. (a) Two electrodes of the capacitive sensor over the plastic IV drip bag filled with medicine $(0.9 \%$ saline). (b) Two electrodes of the capacitive sensor over the plastic IV drip bag filled with air.

pair of electrodes. An influence of the coplanar capacitive sensor to detect the capacitance is the IV drip bag when filled in sodium solution, the surface of the plastic IV drip bag is slightly uneven, but to the naked eye it's completely flat. Also, the copper electrodes of capacitive sensors are always made with ordinary printed circuit board(PCB), then, the electrodes could not attach to the surface of the plastic IV drip bag completely. For this problem solving, use of a flexible PCB is represented in this study. The flexible PCB is extensively used in electronics and micro-system packaging applications. They consist of alter- nating layers of metal and dielectric layers(bulk plastics or polymer composites), each from one 


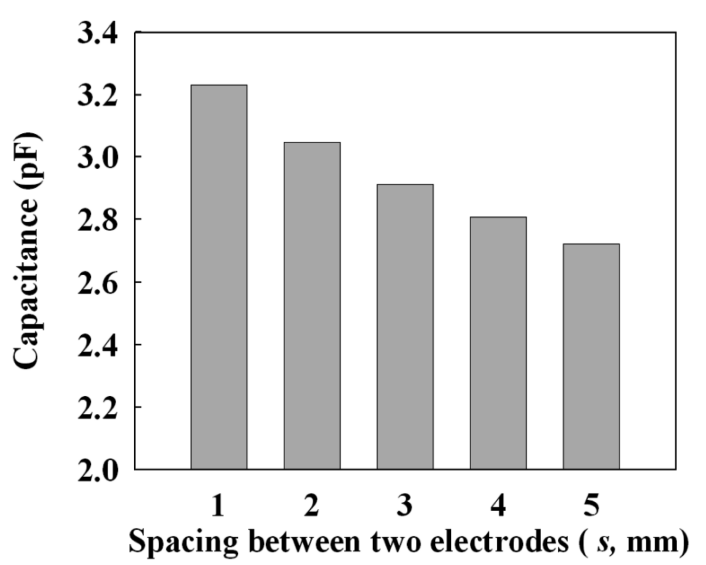

(a)

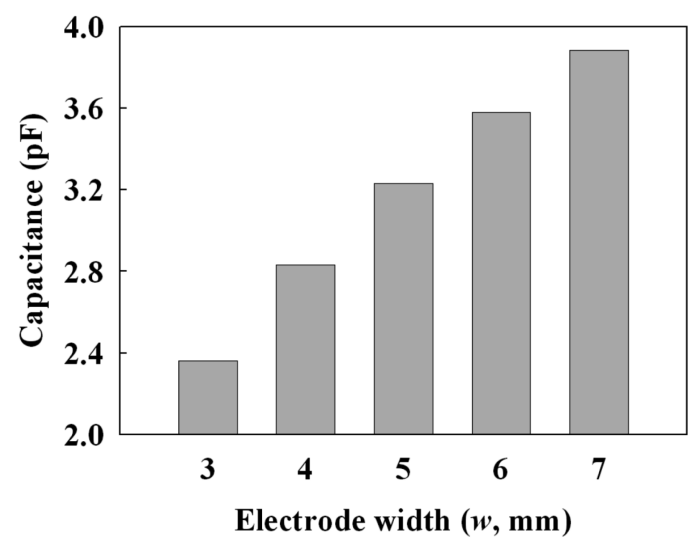

(b)

Fig. 3. The influence of capacitive sensor geometrical directions obtained from the FEA simulation. (a) Capacitance versus electrode width. (b) Capacitance versus spacing between two electrodes.

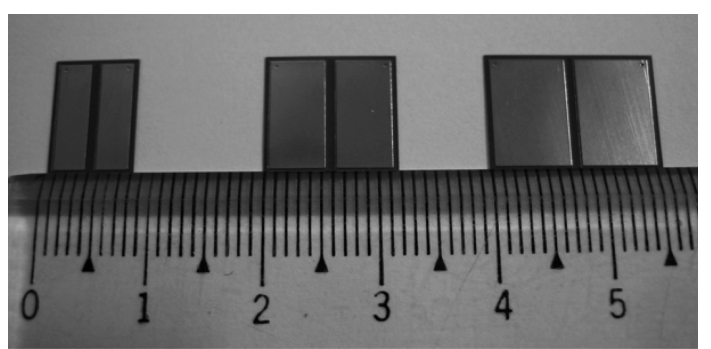

Fig. 4. The implemented capacitive electrodes in 3 sizes made with flexible PCB.

layer up to tens of alternating layers ${ }^{[10]}$. Using the flexible PCB, the electrodes can be attached to the surface of the plastic IV drip bag easily and effectively.

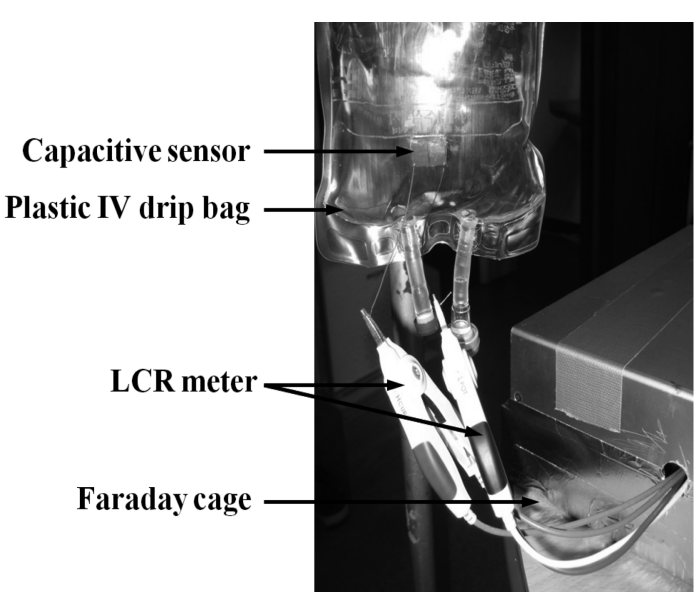

Fig. 5. The capacitive sensor in experiment.

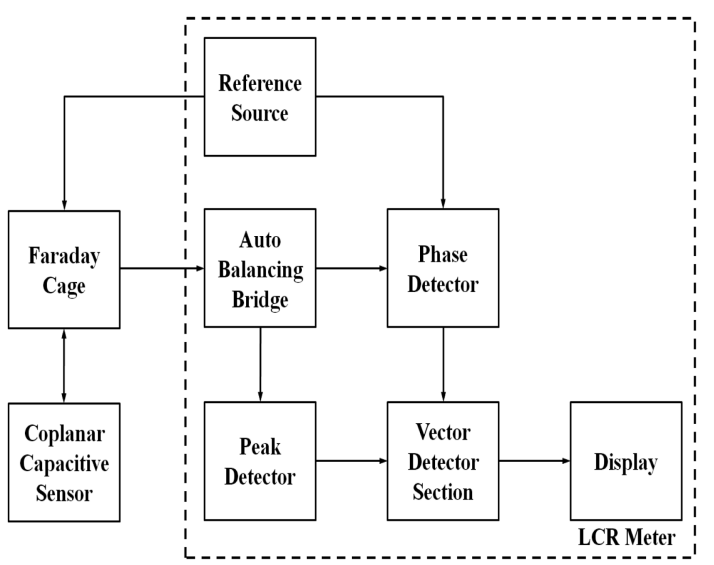

Fig. 6. The diagram of experiment at setting.

\section{Experiment at Validation}

\subsection{Experiment at setup}

Fig. 4. shows 3 kinds of implemented capacitive sensors which were composed of two same-size copper electrodes with flexible PCB. Each electrode is $3 \mathrm{~mm} \times$ $10 \mathrm{~mm}$ (the small sensor), $5 \mathrm{~mm} \times 10 \mathrm{~mm}$ (the mediumsized sensor), and $7 \mathrm{~mm} \times 10 \mathrm{~mm}$ (the large sensor) wide, respectively according to their different types of capacitive sensor. The spacing between two electrodes was set at $1 \mathrm{~mm}$ and thickness of $0.1 \mathrm{~mm}$. Fig. 5 shows the capacitive sensor attached to the bottom surface of the plastic IV drip bag. 3 kinds of capacitive sensors were individually attached to the same place by $3 \mathrm{M}$ glue spray. In the experiment, the plastic IV drip bag was 


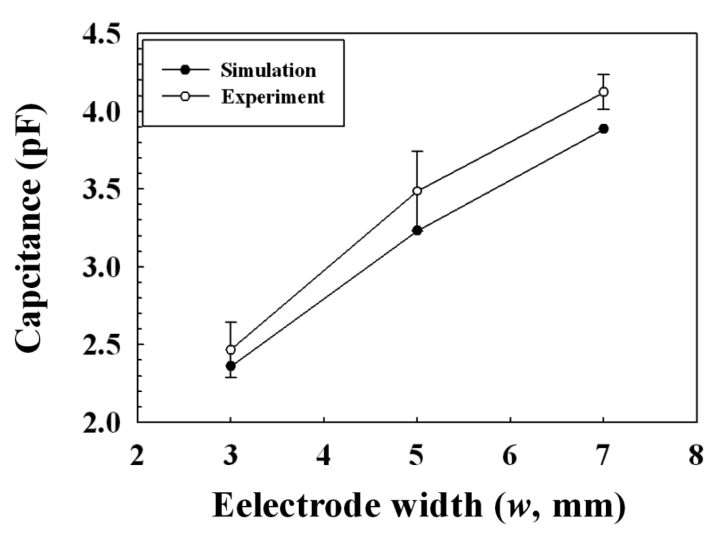

Fig. 7. Capacitance measurements obtained from $t$ experiment and the FEA by 3 kinds of implement capacitive sensors.

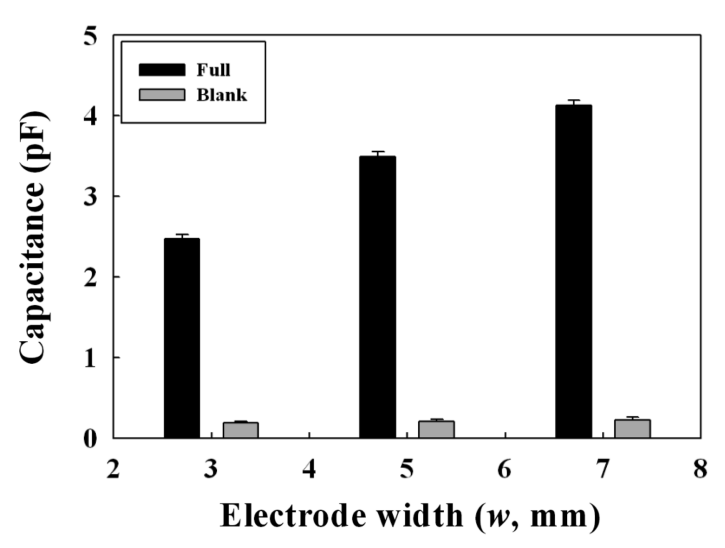

Fig. 8. Capacitance obtained from the full and empty plastic IV drip bag.

filled with $0.9 \%$ saline, the same as the medicine in the FEA simulation. The capacitive sensor was connected to the LCR meter(1061A chroma) to determine its capacitance. The probes of the LCR meter were passed a faraday cage. The faraday cage consisting of solid metal around a noisy circuitry prevents from interfering with the sensitive analog circuitry on the same die ${ }^{[11]}$.

\subsection{Results}

Fig. 7 shows the capacitance measurement obtained from the 3 kinds of implemented capacitive sensors. These experiments were conducted 10 times as they were individually attached to the same place of the same plastic IV drip bag. The capacitance increases as the electrode width increases. The value of capacitance obtained from experiments is illustrated in Fig. 7. A comparison of the capacitance values obtained from the results of experiments and the results of FEA simulation shows a good relationship, and the two results have a difference of $6 \%$. Another comparison of the capacitance measurement of the plastic IV drip bag filled with medicine and was empty is shown in Fig. 8. The values of capacitance measured from the plastic IV drip bag filled with medicine were larger than the plastic bag IV drip that was empty.

The results of the experiment showed comparable results with the estimates from the FEA simulation. The average difference between capacitance value obtained from the experiment and the results of the FEA simulation was assumed to be due to the surface of the plastic IV drip bag was slightly uneven. There is a big difference in results between the plastic IV drip bags filled in medicine and those that are empty. According to the results of the experiment, capacitive sensors can distinguish the plastic bag IV drip with medicine or not, therefore, the idea of using capacitive sensors to detect the residual quantity of the plastic IV drip bag is possible.

\section{Conclusion}

The proposed capacitive sensor to detect the residual quantity of plastic bag IV drip was found to be feasible. The geometrical dimension of the electrodes and spacing between two electrodes of a capacitive sensor were the largest influence on the measurements. The FEA simulation showed that using two electrodes of a capacitive sensor to detect the plastic bag IV drip was feasible. The experiment results showed a good agreement between the experiment and the FEA simulation. They clearly showed the difference between the plastic IV drip bags filled with medicine and those that were empty.

\section{Acknowledgement}

This work was supported by grand No. 10031779 from the Strategic Technology Development Program of Ministry of Knowledge Economy. Also, this work was supported by the Grant of the Korean Ministry of Education, Science and Technology(The Regional Core Research Program/Anti-aging and Well-being Research Center). 


\section{Reference}

[1] http://www.wikipedia.com, U. S. A.

[2] http://www.uhms.org, U. S. A

[3] Y. S. Yang and H. C. Kim, "Development of a miniaturized IV drip rate meter using optical sensors and fuzzy rule based detection algorithm", Proceedings of the 20th Annual International Conference of the IEEE Engin- eering in Medicine and Biology Society, vol. 20, no. 4, pp. 1795-1798, 1998.

[4] D. S. Kang, M. J. Kim, and W. K. Moon, "Optimization of a capacitive sensor for high dynamic range", J. Kor. Sensors Soc., vol 19, pp. 92-98, 2010.

[5] A. A. Nassr, W. H. Ahmed, and W. W. ElD hakhni, "Coplanar capacitance sensors for detecting water intrusion in composite structures", Measurement science and technology, vol. 19, pp. 1-7, 2008.

[6] P. Donald and B. Akhil, "Hyperchloremic metabolic acidosis is a predictable consequence of intraoperative infusion of $0.9 \%$ saline", The Journal of the American Society of Anesthesiologisis, vol. 90 , no. 5, pp. 1247-1249.
[7] Maxwell 2D, V.10 Ansoft Coporation, Pittsburg, 2003.

[8] J. M. Kim, D. H. Oh, J. H. Park, J. W. Cho, Y. W. Kwon, C. Y. Cheon, and Y. K. Kim, "Permittivity measurements up to $30 \mathrm{GHz}$ using micromachined probe", Journal of Micromechanics and Microengineering, vol. 15, pp. 543-550, 2005.

[9] B. H. Brwon, A. Leathard, A. Sinton, F. J. McArdle, R. W. M. Smith, and D. C. Barber, "Blood flow imaging using electrical impedance tomography", Clinical Physics and Physiological Measurement, vol. 13, pp. 175-179, 1992.

[10] H. Yousef, K. Hjort, and M. Lindeberg, "Reliable small via interconnects made of multiple submicron wires in flexible PCB", Journal of Micromechanics and Microengineering, vol. 17, pp. 700-708, 2007.

[11] S. Stefanou, J. S. Hamel, P. Baine, B. M. Armrong, H. S. Gamble, M. Kraft, and H. A. Kemhadjian, "Ultralow silicon substrate noise crosstalk using metal faraday cages in an SOI technology", IEEE transactions on electron devices, vol. 41, no. 3, pp. 486-491, 2004.

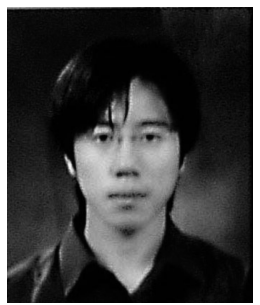

\section{Qun Wei}

- received his B.S. degree in Electrical Engineering from Kyungpook National University, Korea in 2008. He is M.S. candidate in Electrical Engineeing at Kyungpook National University in Korea. His research interests include sensor application and neural engineering.

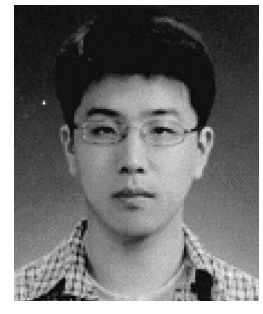

\section{Sang Hyo Woo}

- received his B.S. and M.S. degrees in Electrical Engineering from Kyungpook National University, Korea in 2004 and 2006, respectively. $\mathrm{He}$ is $\mathrm{Ph} . \mathrm{D}$ candidate in Electrical Engineering at Kyungpook National University in Korea. His research interests include telemetry system base on the capsule endoscope. Especially, electrical stimulus and measuring peristalsis movement of the small intestine. 


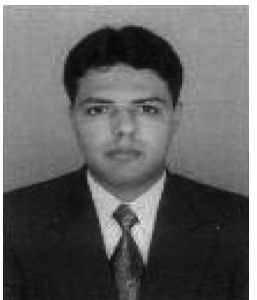

\section{Zia Mohy-Ud-Din}

- received his BS degree in Biomedical Engineering from Sir Syed University, Pakistanin 2005, and received M.S. degree from Medical \& Biological Engineering department, Kyungpook National University, Korea in 2008. $\mathrm{He}$ is $\mathrm{Ph} . \mathrm{D}$ candidate in Medical \& Biological Engineering department at Kyungpook National University in Korea. His research interests include $\mathrm{BCI}$ for emotion detection, biomedical signalprocessing, neuralengineering, and biotele- metry in $\mathrm{BCI}$.

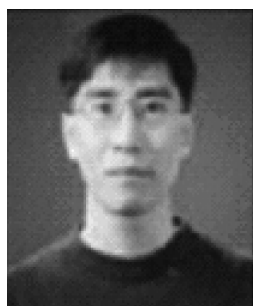

\section{Chul Ho Won}

- received his Ph. D. degree form kyungpook National University, Daegu, Korea, in 1998. From 1999 to 2002, he was post-doctoral research at University of Iowa. He received Stauffer Award for outstanding basic science paper from Academic Radiology journal in 2002. Since 2002, he has been as assistant professor in the dept. of computer control engineering at Kyungil University. His research interests include medical image processing, bio-signal processing.

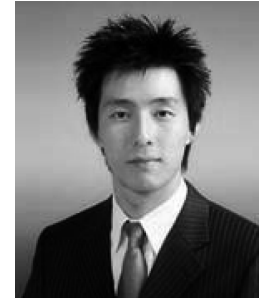

\section{Dong Wook Kim}

- received his B.S. degree in Electrical Engineering from Inje University, Korea in 2007, and received his M.S. degree in Electrical Engineering from Kyungpook National University, Korea in 2009. He is Ph.D candidate in Electrical Engineering at Kyungpook National University in Korea. His research interests include bidirectional hearing aids and sound signal processing.

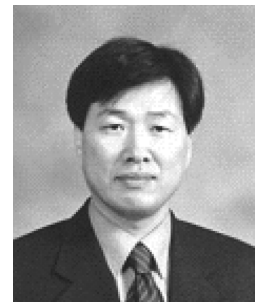

\section{Jin Ho Cho}

- received his $\mathrm{Ph} . \mathrm{D}$. degree from $\mathrm{Ky}-$ ungpook National University in 1988. He was a visiting professor in dept. of Biomedical engineering, College of Engineering at University of Iowa, USA, in 1991. He is Professor in the school of Electrical Engineering, College of IT Engineering, Kyungpook National University, a vice chairman of at the Korea Society of Medical Biological Engineering, a Director of the Advanced Research Center of Recovery of Human Sensibility. His research interests include the biomedical signal processing, sensor applications, and biomedical instrumentations. He is a Member of the IEEE. 